\title{
IoT device management using semantics for distinguishing device compatibility
}

\begin{abstract}
The main objective of this paper is to achieve the device compatibility between multiple devices which are defined in the ontology. For example if a temperature sensor D1 wants to communicate with the actuator fan D2 due to their different data formats and defined properties they are unable to communicate with each other. The interlinking of data derived from different devices used to create semantics for interoperation with a shared meaning. So we are trying to solve the device compatibility problem by using their semantics. To achieve the semantic interoperability we described a ontology module. In this paper we proposed an ontology model using semantics for distinguishing device capability and also the query execution time for the devices. This ontology model is designed for adaptable IoT systems.
\end{abstract}

